\title{
Neuroendocrine carcinoma of the breast with a mucinous carcinoma component: A case report with review of the literature
}

\author{
MITSUAKI ISHIDA $^{1}$, TOMOKO UMEDA ${ }^{2}$, HAJIME ABE $^{2}$, TOHRU TANI ${ }^{2}$ and HIDETOSHI OKABE ${ }^{1}$ \\ ${ }^{1}$ Department of Clinical Laboratory Medicine and Division of Diagnostic Pathology; \\ ${ }^{2}$ Department of Surgery, Shiga University of Medical Science, Otsu, Shiga 520-2192, Japan
}

Received January 30, 2012; Accepted March 30, 2012

DOI: $10.3892 / \mathrm{ol} .2012 .678$

\begin{abstract}
Neuroendocrine carcinoma (NEC) of the breast is a rare distinct clinicopathological entity, which, clinically, tends not to be aggressive. Mucinous carcinoma of the breast is also a rare distinct entity and is classified into types $A$ (paucicellular) and B (hypercellular). It is well known that type B mucinous carcinoma frequently shows neuroendocrine differentiation. However, the coexistence of NEC and mucinous carcinoma within the same breast tumor is extremely rare. In the present study, we report a case of solid NEC of the breast with a mucinous carcinoma component and discuss the tumorigenesis of this extremely rare lesion. A 37-year-old Japanese female presented with a right breast tumor. Mastectomy and removal of the right axillary lymph nodes were performed. The resected breast tumor was composed of solid NEC (approximately 85\% of the tumor) and type B mucinous carcinoma components. The right axillary lymph nodes had metastatic solid NEC. Three years later, local recurrence of mucinous carcinoma occurred in the operation scar of the right thoracic wall. In addition, seven years after the first surgery, metastatic solid NEC in the liver was observed. Recent molecular studies clearly revealed that no differences in gene expression are present between type B mucinous carcinoma and NEC. Therefore, the present case may represent solid NEC and type B mucinous carcinoma as part of a spectrum with the same genetic background. Moreover, the prognosis of solid NEC is thought to be good, and metastasis is extremely rare. However, metastasis of solid NEC of the breast may occur at a later stage. Therefore, long-term follow-up is required for patients with solid NEC of the breast.
\end{abstract}

Correspondence to: Dr Mitsuaki Ishida, Department of Clinical Laboratory Medicine and Division of Diagnostic Pathology, Shiga University of Medical Science, Tsukinowa-cho, Seta, Otsu, Shiga 520-2192, Japan

E-mail: mitsuaki@belle.shiga-med.ac.jp

Key words: neuroendocrine carcinoma, mucinous carcinoma, breast

\section{Introduction}

Neuroendocrine carcinoma (NEC) of the breast is a rare distinct clinicopathological entity, comprising $0.5-2 \%$ of breast carcinomas worldwide $(1,2)$. Since previous diagnostic criteria for NEC of the breast had been contradictory, the World Health Organization Classification of Tumours of the Breast and Genital Organs proposed the following diagnostic criteria for breast NEC in 2003: i) Presence of morphological neuroendocrine features resembling those of neuroendocrine tumors of the gastrointestinal tract and lung, and ii) expression of neuroendocrine markers in more than 50\% of tumor cells (1).

Mucinous carcinoma of the breast is also a rare distinct clinicopathological entity, and accounts for approximately $2 \%$ of breast carcinomas worldwide (1). It is characterized by a proliferation of clusters of generally small and uniform tumor cells floating in large amounts of extracellular mucus. Pure mucinous carcinoma is not recognized as a single homogenous entity. Capella et al classified it based on structural and cytological features as type A (paucicellular; a tumor showing a ribbon, annular, or cribriform growth pattern with prominent extracellular mucin) and type B (hypercellular; tumor showing clumps or sheet-like structures with less extracellular mucin) (3). It is well known that type B mucinous carcinoma frequently shows neuroendocrine differentiation $(3,4)$.

Solid NEC of the breast with a mucinous carcinoma component is rarely reported (2). In the present study, we report a case of solid NEC of the breast with a mucinous carcinoma component and discuss the tumorigenesis of this rare lesion.

The study was approved by the ethics committee of the university and patient consent was obtained.

\section{Patients and methods}

Case report. A 37-year-old Japanese female presented with a right breast tumor. A physical examination revealed a relatively well-circumscribed tumor, measuring $3 \times 2.5 \mathrm{~cm}$ in diameter, in the right breast and swollen right axillary lymph nodes. No metastatic lesions with the exception of the right axillary lymph nodes were detected by computed tomography (CT). The biopsy specimen of the right breast tumor revealed invasive carcinoma; thus, total mastectomy and removal of the right axillary lymph nodes were performed (cT2N1M0, stage IIB). Chemotherapy and hormonotherapy were adminis- 


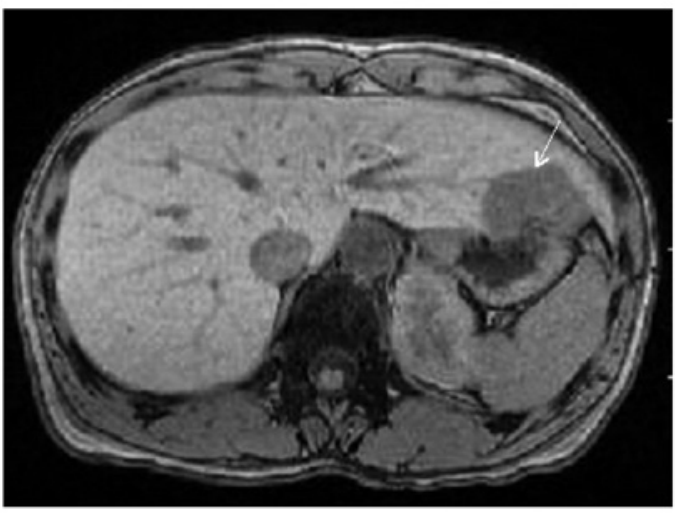

Figure 1. Magnetic resonance imaging showing a relatively well-circumscribed tumor in the liver (arrow).

tered following the surgery. Three years later, local recurrence was observed on the operation scar of her right thoracic wall, and tumor resection was performed again. Seven years after the first surgery, a liver tumor was detected during the follow-up CT. CT and magnetic resonance imaging demonstrated a relatively well-circumscribed tumor, measuring 42x37x30 mm, in S3 (Fig. 1). Metastatic breast cancer in the liver was suspected clinically, resulting in the surgical resection of the liver tumor. The post-operative course was uneventful, and the patient has been free from recurrence for 18 months of medical follow-up.

Methods. The formalin-fixed, paraffin-embedded tissue blocks of the tumors of the breast, local recurrence on the operation scar, and liver were cut into $3-\mu \mathrm{m}$ sections, deparaffinized and rehydrated. Each section was stained with hematoxylin and eosin, and then used for immunostaining. Immunohistochemical analyses were performed using an autostainer (XT system Benchmark, Ventana Medical System, Tucson, AZ, USA) according to the manufacturer's instructions. The primary antibodies used were: a mouse monoclonal antibody against CD56 (clone CD564, Novocastra Laboratories, Ltd., Newcastle upon Tyne, UK), a mouse monoclonal antibody against chromogranin A (clone DAK-A3, Dako Cytomation, Glostrup, Denmark), a mouse monoclonal antibody against estrogen receptor (ER) (clone 6F11, Novocastra), a mouse monoclonal antibody against gross cystic disease fluid protein-15 (GCDFP15) (clone 23A3, Novocastra), a mouse monoclonal antibody against progesterone receptor (PgR) (clone PgR636, Dako), and a mouse monoclonal antibody against synaptophysin (clone 27G12, Novocastra). In addition, immunohistochemistry for the $c-e r b B-2$ (HER2) oncoprotein was performed using a Dako kit.

\section{Results}

Breast tumor. The tumor was relatively well-circumscribed, however, it had invaded into the surrounding fatty tissue. Most of the tumor comprised variable-sized solid nests with or without central necrosis separated by delicate fibrovascular stroma (Fig. 2A and B). The solid nests consisted of uniform tumor cells which had slightly enlarged oval nuclei and inconspicuous nucleoli with slightly eosinophilic cyto-
A

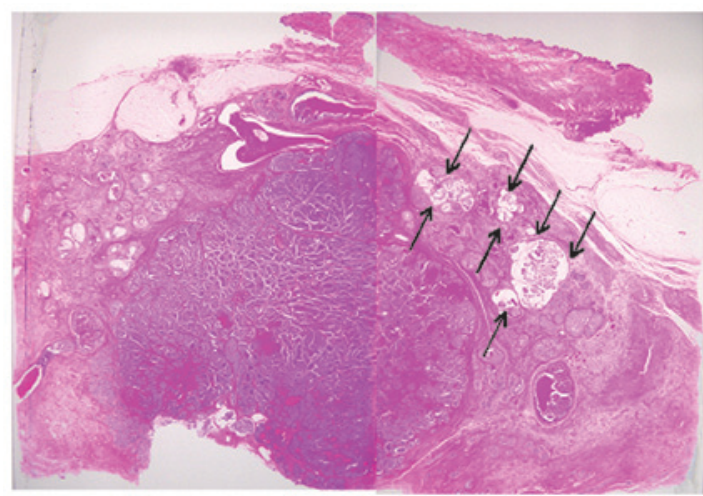

B

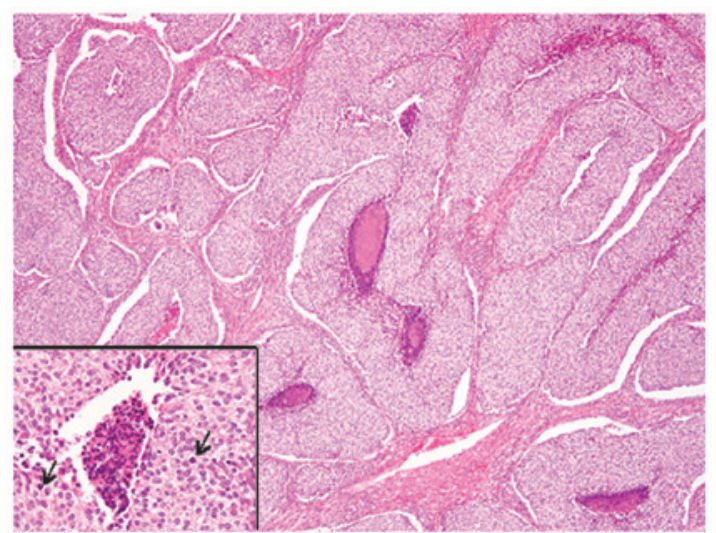

C

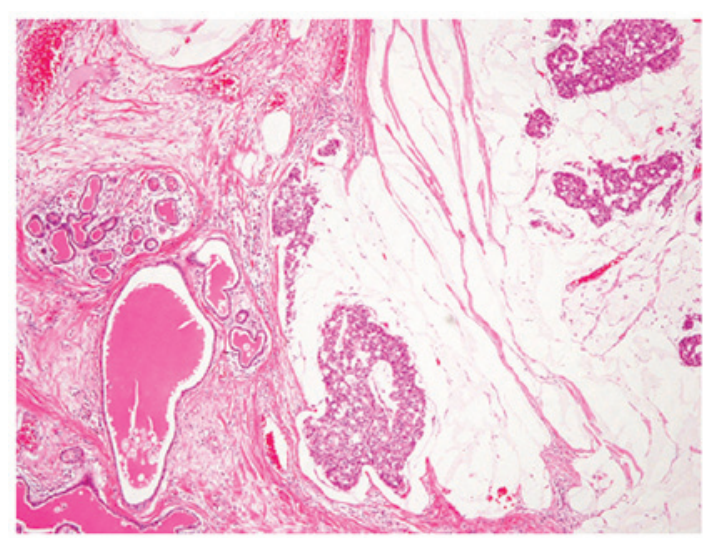

Figure 2. Histopathological features of the right breast tumor (H\&E stain). (A) Panoramic view of the breast tumor demonstrates invasive growth forming solid nests. At the periphery of the tumor, mucinous carcinoma component is present (arrows). (B) Neuroendocrine carcinoma of the breast. Variable-sized solid nests with or without central necrosis. Tumor cells have mildly enlarged nuclei and slightly eosinophilic cytoplasm. Mitotic figures are scattered (arrows, inset). Original magnification, x40 (inset, x200). (C) Mucinous carcinoma component. Sheets of tumor cells are floating in the extracellular mucin. Original magnification, x100.

plasm (Fig. 2B, inset). Mitotic figures were easily detected (45/10 high power fields).

Immunohistochemical analyses revealed that these tumor cells were diffusely ( $>95 \%$ of the tumor) positive for chromogranin A (Fig. 3), although CD56, synaptophysin and GCDFP-15 were not expressed. Therefore, this component was considered to be solid NEC of the breast. In addition, ER and PgR were diffusely expressed (100\% of the tumor), and the HER2 score was $1+$. 


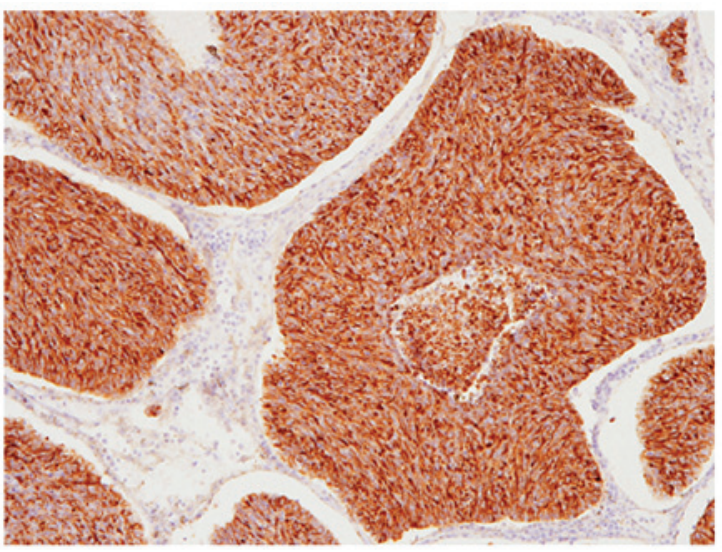

Figure 3. Immunohistochemical finding in the right breast tumor. Chromogranin A is diffusely expressed in the tumor cells of the neuroendocrine carcinoma. Original magnification, x100.

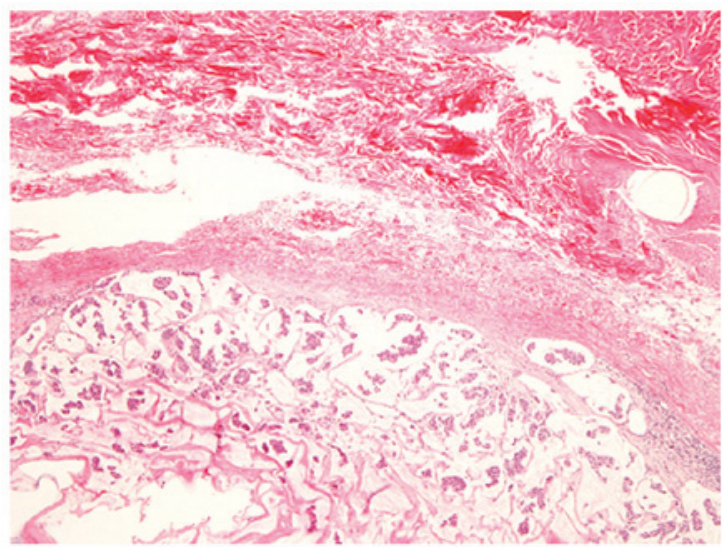

Figure 4. Local recurrence of the mucinous carcinoma in the operation scar of the right thoracic wall (H\&E stain). Original magnification, $\mathrm{x} 40$.

Approximately $85 \%$ of the tumor belonged to the abovementioned solid NEC component. However, areas with proliferation of clusters or sheets of uniform tumor cells with mildly enlarged nuclei and slightly eosinophilic cytoplasm floating in extracellular mucin, which are characteristic histopathological features of mucinous carcinoma, were present at the periphery of the tumor (Fig. 2A and C). According to the classification by Capella et al (3), this mucinous carcinoma was classified as type B. Results of the immunohistochemical analysis revealed that certain tumor cells of the mucinous carcinoma component were positive for chromogranin A, but negative for CD56, synaptophysin and GCDFP-15. In addition, ER and PgR were diffusely expressed (100\% of the tumor) in this mucinous carcinoma component and the HER2 score was 0.

According to these histopathological and immunohistochemical findings, an ultimate diagnosis of solid NEC with type B mucinous carcinoma component was made. In addition, the right axillary lymph nodes had metastatic solid NEC (3/28).

Local recurrent tumor. The tumor was located in the dermis to superficial subcutis and was composed of clusters of uniform tumor cells with slightly enlarged nuclei floating in extracel-
A

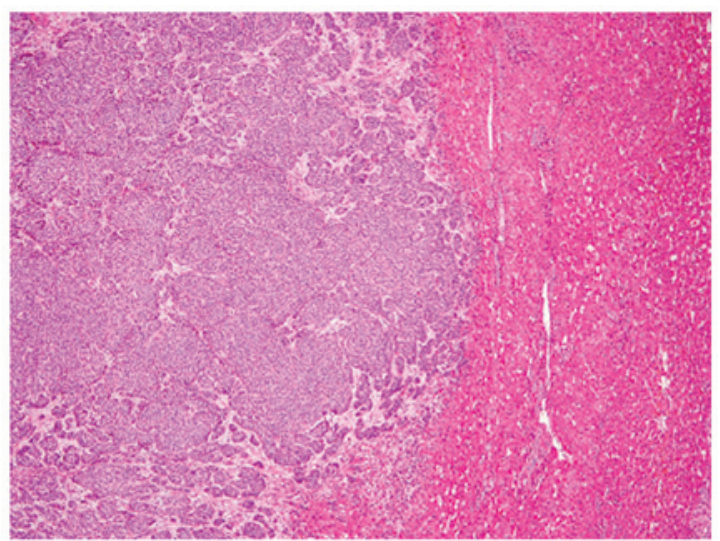

B

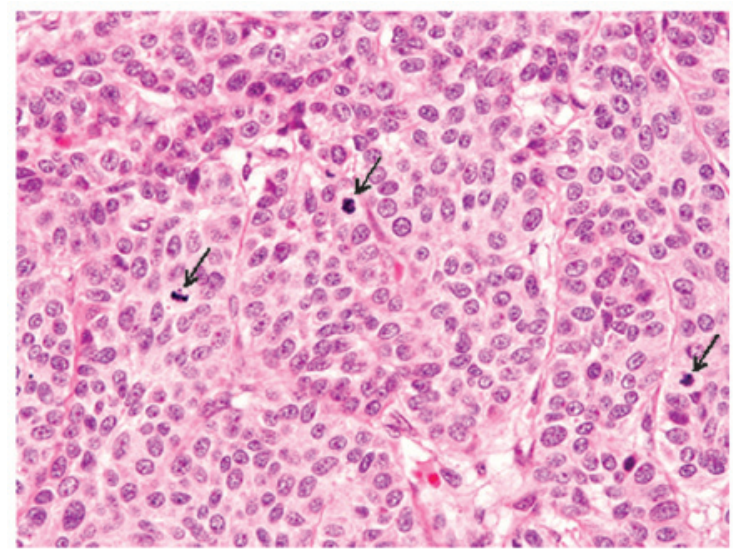

Figure 5. Histopathological features of the liver tumor (H\&E stain) (A) Solid nests or sheets of tumor cells in the liver. Original magnification, $\mathrm{x} 40$ (B) Tumor cells have mildly enlarged nuclei and slightly eosinophilic cytoplasm. Mitotic figures are scattered (arrows). Original magnification, x200.

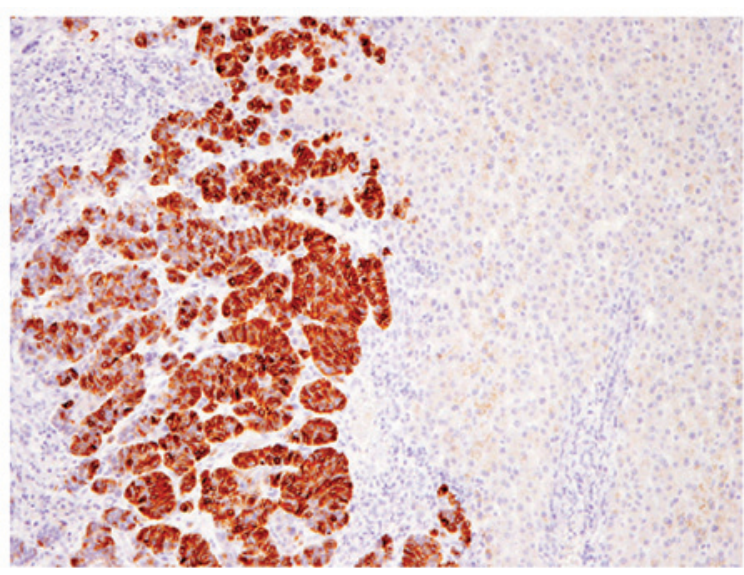

Figure 6. Immunohistochemical finding in the liver tumor. Tumor cells diffusely expressing chromogranin A (left: tumor cells, right: liver parenchyma). Original magnification, $\mathrm{x} 100$.

lular mucin (Fig. 4). These histopathological features were consistent with local recurrence of mucinous carcinoma of the breast. No NEC component was present.

Liver tumor. The tumor was relatively well-circumscribed from surrounding liver tissue and separated by delicate fibrovascular 
stroma (Fig. 5A). The tumor was composed of solid nests of slightly enlarged oval nuclei with inconspicuous nucleoli and slightly eosinophilic cytoplasm (Fig. 5B). Mitotic figures were easily detected (47/10 high power fields).

Immunohistochemical analyses revealed that these tumor cells were diffusely (>95\% of the tumor) positive for chromogranin A (Fig. 6), although CD56, synaptophysin and GCDFP-15 were not expressed. No mucinous carcinoma component was present. Therefore, a diagnosis of metastatic solid NEC of the breast in the liver was made. In addition, ER and PgR were diffusely expressed (100\% of the tumor) and the HER2 score was 0.

\section{Discussion}

According to the WHO Classification, NEC of the breast is classified into three subtypes: solid neuroendocrine carcinoma, small cell/oat cell carcinoma, and large cell neuroendocrine carcinoma (1). The histopathological features of the present case corresponded to solid NEC of the breast, which is the most common subtype (5), since the tumor was composed of solid nests with or without central necrosis separated by delicate fibrovascular stroma, which suggested neuroendocrine differentiation, and no poorly differentiated component was observed. López-Bonet et al summarized the clinicopathological and immunohistochemical features of seven cases of solid NEC of the breast (2). Results of their study showed that solid NEC of the breast mainly affects the elderly (the median age of the patients was 63), and the frequency of axillary lymph node metastasis is relatively high (3/6 cases underwent lymph node removal) (2). Immunohistochemically, ER and PgR were positive in all cases, as in the present case (2), and overexpression of HER2 is uncommon in NEC of the breast $(2,5)$. Moreover, the expression pattern of neuroendocrine markers is variable; all cases reported by López-Bonet et al demonstrated positive immunoreactivity for synaptophysin ( $>50 \%$ of tumor cells), but chromogranin A expression was observed only focally in five of their seven cases (the remaining two cases demonstrated no positive immunoreactivity for chromogranin A) (2). By contrast, Sapino et al reported that $53 \%$ of NEC expressed chromogranin A ( $>50 \%$ of tumor cells) (6).

A noteworthy finding of the present case is the coexistence of type B mucinous carcinoma and solid NEC within the same breast tumor. Although mucinous carcinoma (particularly type B) frequently shows neuroendocrine differentiation, the presence of a dual (neuroendocrine and mucinous) divergent differentiation within the same breast tumor is extremely rare (5). López-Bonet et al reported two cases of solid NEC with a mucinous carcinoma component (the subtype of the mucinous carcinoma is not available) (2). Thus, this is the third reported case of solid NEC with mucinous carcinoma component of the breast.

Recently, Weigelt et al analyzed the molecular characteristics of mucinous carcinoma and NEC of the breast using genome-wide oligonucleotide microarrays (7). The study clearly revealed that no differences in gene expression were present between type B mucinous carcinoma and NEC, whether or not type A mucinous carcinoma exhibited differences compared with type B mucinous carcinoma and NEC (7). Taking these results into consideration, the present case may represent NEC and type B mucinous carcinoma as part of a spectrum with the same genetic background.

The prognosis of solid NEC is thought to be better than invasive ductal carcinoma, since in one report, none of the investigated solid NEC (35 cases) had distant metastasis (5). In another study, only one of seven cases of solid NEC demonstrated metastasis (soft tissue of the cheek; the patient is alive with metastatic disease) (2). In addition, both patients with solid NEC with mucinous carcinoma component reported by López-Bonet et al are free from tumor recurrence (2). However, the present patient had liver metastasis seven years after the surgery. Therefore, long-term follow-up is necessary for patients with solid NEC of the breast due to metastatic potential at a later stage.

\section{References}

1. Ellis IO, Schnitt SJ, Sastre-Garau X, et al: Invasive breast carcinoma. In: World Health Organization of Classification of Tumors. Pathology and Genetics of Tumours of the Breast and Female Genital Organs. Tavassoli FA and Devilee P (eds) IARC Press, Lyon, pp30-34, 2003.

2. López-Bonet E, Alonso-Ruano M, Barraza G, Vazquez-Martin A, Bernadó L and Menendez JA: Solid neuroendocrine breast carcinomas: Incidence, clinico-pathological features and immunohistochemical profiling. Oncol Rep 20: 1369-1374, 2008.

3. Capella C, Eusebi V,Mann B and Azzopardi JG: Endocrine differentiation in mucoid carcinoma of the breast. Histopathology 4: 613-630, 1980.

4. Scopsi L, Andreola S, Pilotti S, et al: Mucinous carcinoma of the breast: a clinicopathologic, histochemical, and immunocytochemical study with special reference to neuroendocrine differentiation. Am J Surg Pathol 1994: 702-711, 1994.

5. Righi L, Sapino A, Marchió C, Papotti M and Bussolati G: Neuroendocrine differentiation in breast cancer: established facts and unresolved problems. Semin Diagn Pathol 27: 69-76, 2010.

6. Sapino A, Righi L, Cassoni P, Papotti M, Gugliotta P and Bussolati G: Expression of apocrine differentiation markers in neuroendocrine breast carcinomas of aged women. Mod Pathol 14: 768-776, 2001.

7. Weigelt B, Geyer FC, Horlings HM, Kreike B, Halfwerk H and Reis-Filho JS: Mucinous and neuroendocrine breast carcinomas are transcriptionally distinct from invasive ductal carcinomas of no special type. Mod Pathol 22: 1401-1414, 2009. 